\title{
Measurements of Amorphous (a)-C, Au + a-C and a-Metal TEM Specimen Stabilities for HRTEM Studies
}

\author{
Rodney A. Herring ${ }^{1 *}$ \\ 1. CAMTEC, MENG, University of Victoria, British Columbia V8W 2Y2, Canada \\ * Corresponding author: rherring@uvic.ca
}

The stability of modern aberration-corrected electron microscopes operating in a highly quiescent environment have become so stable that their spatial resolution measurements often depend on the stability of the specimen [1]. A Fourier transform of a high-resolution image is probably the easiest and most often applied method to see the information content, however, its dependence on the specimen's stability suffers from two faults. If the specimen is inherently unstable due to irradiation damage, beam heating and atomic migrations and rearrangements such as seen with amorphous-carbon (a-C) specimens there is a loss of beam coherence resulting in a low estimate of the microscope's spatial resolution. If the specimen is a stable crystal such as gold ( $\mathrm{Au}$ ) that doesn't suffer from the inherent instabilities associated with a-C, the crystal's low-frequency Fourier peak intensities contribute to the higher Fourier peak intensities resulting a high estimate of the microscope's spatial resolution. A mixed amorphous/crystal specimen such Au coated a-C suffers from both inherent instabilities and the Fourier transform extension of the Fourier intensities. Required for a better measurement of the microscope's spatial resolution is a stable amorphous specimen. Often amorphous tungsten, a heavy atom but soft metal, is used for this purpose but it recrystallizes while under the beam so is unstable during TEM observation especially at the higher voltages. Thus, the search for a better high-resolution TEM specimen was conducted. Amorphous metal (a-M) such as Zr65.7 - Cu5.6 - Ni11.8 - Al3.7 - Ti3.3 (wt\%) consists of heavy atoms locked in place and are extremely strong (1524 MPa), both good qualities for a stable specimen. Reported here is a study conducted to compare the TEM specimen stabilities of a-C, $\mathrm{Au}$ coated $\mathrm{a}-\mathrm{C}$ and $\mathrm{a}-\mathrm{M}$ using the Young fringe method of electron beam interference Fourier transformed to reveal their information content [2].

For this study, a Scanning Transmission Electron Holography Microscope (STEHM, Hitachi HF3300v) operating in its TEM mode having an aplanatic aberration corrector (CEOS's bcorr) was aligned before conducting this experiment to provide a phase plate showing a flat phase extending slightly greater than $26 \mathrm{mrad}$ (Fig.1). Young images were acquired having a tilt frequency of $8 \mathrm{~Hz}$ and then Fourier transformed to reveal their information content. The extension of information for the a-C specimen was $9.77 \pm 0.441 / \mathrm{nm}$ (Fig. $2 \mathrm{a}$ ) and for the Au coated a-C specimen was $10.69 \pm 0.411 / \mathrm{nm}$ (Fig. 2b), which showed the Au Fourier peaks extending the Young fringes of the amorphous intensity of the a-C part of the specimen to higher frequencies. The extension of information for the a-M specimen was $17.03 \pm 0.241 / \mathrm{nm}$ (Fig. 2c), the best of the three revealing its higher stability than the other TEM specimen. A previous study of an Au single crystal specimen produced an extension of information to $281 / \mathrm{nm}$ that would have been strongly enhanced by the low order Fourier peaks contributing to the high order Fourier peaks revealing the excellent stability of the STEHM.

Grants from UVic, NSERC Discovery, Engage and CRD, CFI and BCKDF and provision of the a-M samples from Stephanie C. O’Keeffe, Liquidmetal Technologies, Inc. are appreciated. 
References:

[1] D Van Dyck and AF De Jong, Ultramicroscopy. 47 (1992) 266.

[2] J Frank, Optik 44 (1976) 379.

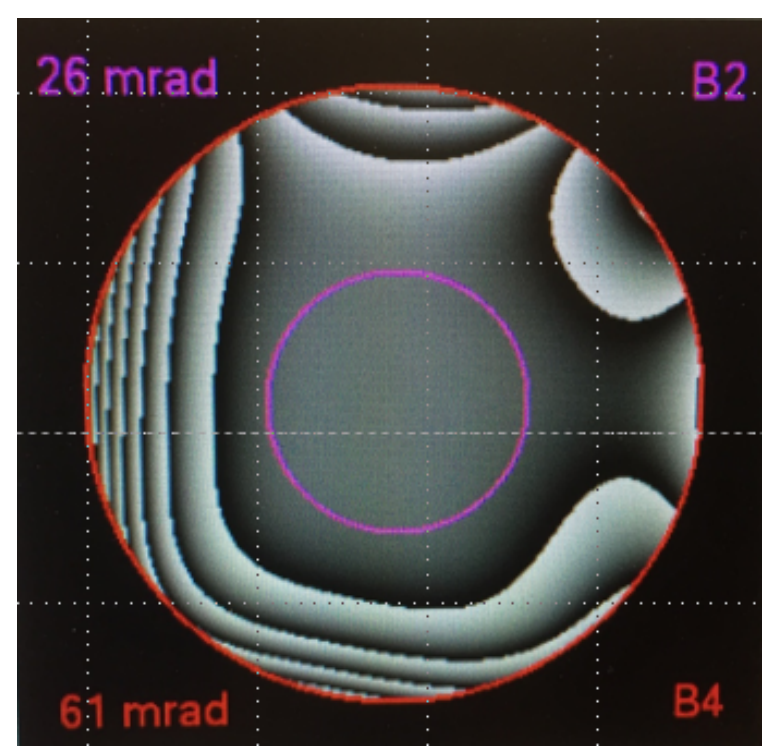

Figure 1. Phase plate showing the alignment of the STEHM for conducting this experiment.
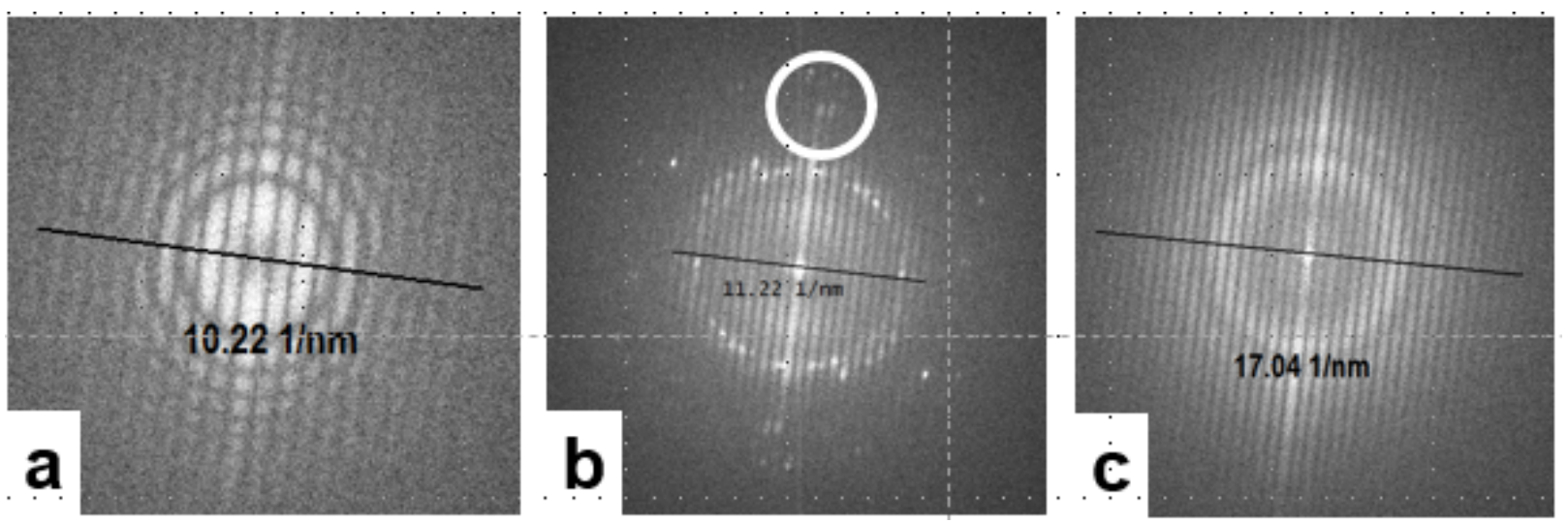

Figure 2. Fourier transforms of Young interference patterns revealing spatial information for a) a-C specimen, b) Au coated a-C specimen and c) a-M specimen indicative of the stability of the specimen showing a-M to be the most stable. The a-c specimen transferred the least amount of information. The Au crystal's peak spatial frequencies can be seen to extend the young fringes to higher frequencies as seen within the circle extending its fringes to higher frequencies than the diffuse a-C intensity. The a-M specimen transferred information to the highest spatial frequencies. 